\title{
ISO Observations of Be Stars
}

\author{
L.B.F.M. Waters
}

Astronomical Institute, University of Amsterdam, Kruislaan 403, NL-1098 SJ Amsterdam, The Netherlands

Instituut voor Sterrenkunde, Katholieke Universiteit Leuven, Celestijnenlaan 200B, B-3001 Heverlee, Belgium

\section{N.R. Trams}

Integral Science Operations Centre, ESTEC, P.O. Box 299, 2200 AG Noordwijk, The Netherlands

S. Hony, A. de Koter, P.W. Morris, and P.A. Zaal

Astronomical Institute, University of Amsterdam, Kruislaan 403, NL-1098 SJ Amsterdam, The Netherlands

\section{J.M. Marlborough and C.E. Millar}

Department of Physics and Astronomy, University of Western Ontario, London, Ontario N6A $3 K 7$, Canada

B. Vandenbussche

Instituut voor Sterrenkunde, Katholieke Universiteit Leuven, Celestijnenlaan 200B, B-3001 Heverlee, Belgium

\begin{abstract}
We discuss the infrared spectral energy distribution of $\mathrm{Be}$ stars, focusing on new results obtained with the Infrared Space Observatory. The 60-160 $\mu \mathrm{m}$ flux of some Be stars is higher than expected, which may be due to cold dust or an outer disk component with enhanced densities. The infrared spectrum of Be stars is dominated by numerous HI recombination lines, whose line strengths show a complex behaviour. The electron temperature in the disk of $\gamma$ Cas was found to be about $9500 \mathrm{~K}$, and evidence for an elevated temperature near the upper part of the disk is presented. Be stars may be recognized from their infrared spectrum on the basis of $\mathrm{HI}$ line flux ratios.
\end{abstract}

\section{Introduction}

Circumstellar material near hot, massive stars is ubiquitous and remains present throughout their entire life. While in young massive stars material from the parental molecular cloud is the main source of circumstellar material, during the main sequence and post-main-sequence life a stellar wind provides the material. Since these stellar winds are of importance for our understanding of massive star 
evolution, quantitative studies of the mass loss rate and of the geometry of the wind are useful to constrain theoretical models for the outflows from hot stars and their impact on stellar evolution.

Be stars are $B$ type dwarfs or giants characterized by the presence (either currently or in the past) of $\mathrm{H} \alpha$ emission in the optical spectrum. The width of the $\mathrm{H} \alpha$ emission correlates with the projected rotational velocity of the stellar photosphere (vsini), and often the profiles are double-peaked (see e.g. Dachs et al. 1986). At infrared and radio wavelengths the continuum energy distribution of $\mathrm{Be}$ stars is characterized by excess radiation above the photosphere due to free-free and free-bound emission from the ionized circumstellar gas (Gehrz et al. 1974; Waters 1986). The optical continuum radiation shows linear polarisation at the 1-2 per cent level (e.g. McLean \& Brown 1978; Quirrenbach et al. 1997; see also K.S. Bjorkman, these proc.), caused by free electrons that scatter stellar radiation. These observations are consistent with a disk-like geometry of the circumstellar ionized gas. Direct proof of the highly flattened geometry of $\mathrm{Be}$ star envelopes has come from imaging at radio (Dougherty \& Taylor 1992) and optical (e.g. Stee et al. 1995, 1998; Quirrenbach et al. 1997) wavelengths.

Despite considerable efforts in modeling the envelopes of Be stars (see e.g. the contributions of J. Bjorkman, Owocki \& Cohen, and Okazaki, these proc.), the origin of the disks is still not well understood. Models for the origin of Be star disks may be constrained if the disk structure is better understood. This can be done by studying the infrared part of the spectrum, both using photometry and spectroscopy. At these wavelengths, the emission from the stellar photosphere is weak, and any radiation from the gas in the disk is readily observable. In addition, the infrared wavelength region contains many spectral lines of hydrogen, helium and other elements that can give clues to the temperature, density and velocity structure of the disk.

This review focusses on recent results obtained with the Infrared Space Observatory (ISO; Kessler et al. 1996), whose instruments allowed for the first time quantitative spectroscopy in the 2-200 $\mu \mathrm{m}$ wavelength region unhampered by the effects of the Earth's atmosphere. The paper is organized as follows: in Sect. 2 we briefly discuss the IR emission from ionized circumstellar gas; Sect. 3 describes new ISO photometry, and Sect. 4 ISO spectroscopy.

\section{IR emission from ionized gas}

At infrared wavelengths, thermal free-free and free-bound emission from the ionized circumstellar gas begin to dominate the flux observed from Be stars. When the emission is optically thin, the excess is directly proportional to the emission measure EM of the gas, defined as $\int n_{e}^{2} d V$. Since the optical depth is proportional to $\rho^{2} \lambda^{2}$, at longer infrared wavelengths the inner parts of the disk will become optically thick. The spectrum will turn down and its wavelength dependence will depend on the radial density structure in the envelope (see e.g. Wright \& Barlow 1975), and, to a lesser degree, on the temperature gradient in the disk (Cassinelli \& Hartmann 1979). The effect of bound-free transitions will be to cause a jump in the spectrum at the series edge. Such a jump can either be in emission or absorption, depending on the temperature structure and geometry of the envelope. 

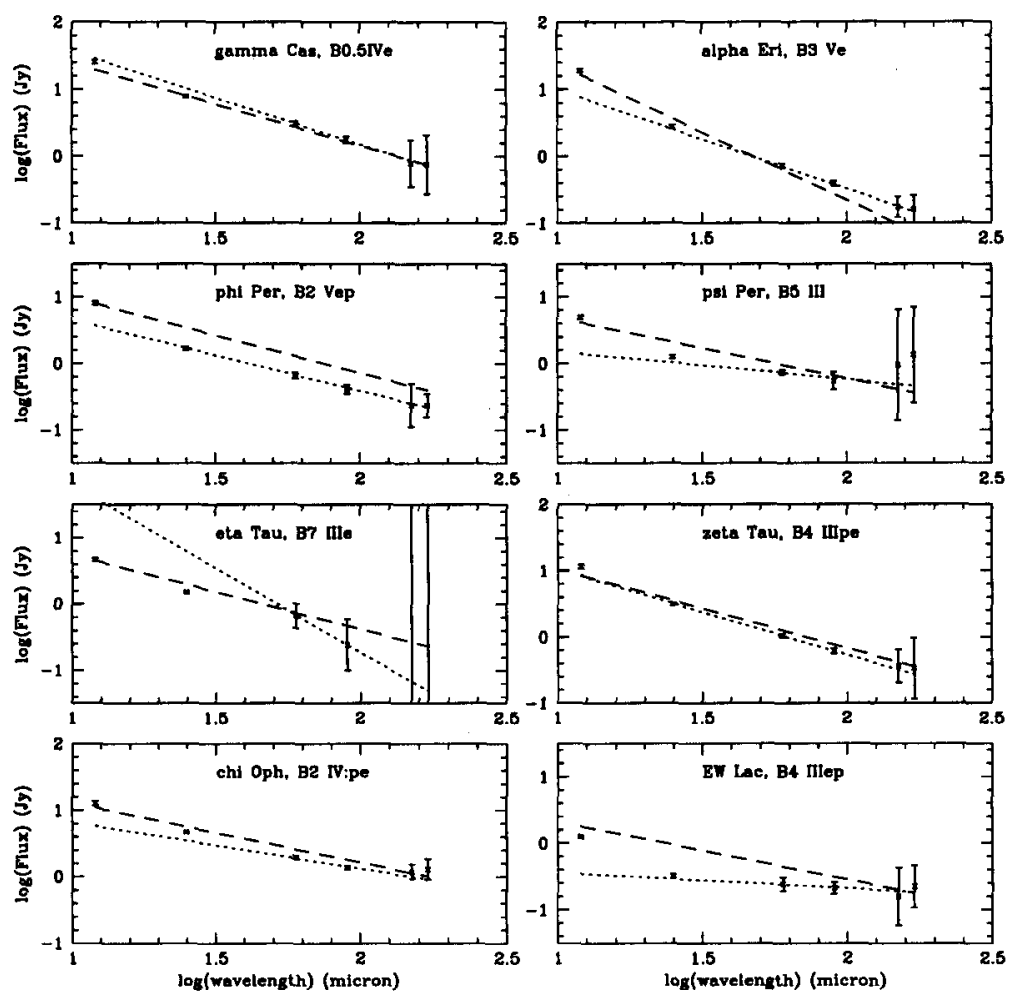

Figure 1. ISO-PHOT continuum energy distributions of eight bright Be stars. The dotted line is a fit to the $60-160 \mu \mathrm{m}$ data points. The dashed line is a fit to the IRAS data points (not shown individually in this plot).

Observations of $\mathrm{Be}$ stars at infrared and radio wavelengths confirm this basic picture (e.g. Gehrz et al. 1974; Dachs \& Wamsteker 1982; Ashok et al. 1984; Waters et al. 1987; Dougherty et al. 1994). At sub-millimeter and radio wavelengths however, the flux from Be stars usually is (much) weaker than expected on the basis of shorter wavelength observations (Taylor et al. 1990; Waters et al. 1991). This turn-down in the spectrum is not well understood, and may be related to a change in geometry or in degree of ionisation in the outer regions of the disk. We will return to this point in the next section.

Apart from continuum radiation, the ionized circumstellar gas will also emit recombination radiation (Persson \& McGregor 1985; Chalabaev \& Maillard 1985; Hamann \& Simon 1987; Sellgren \& Smith 1992; Hony et al. 2000; Rinehart et al. 2000). For the densities that prevail in the gas surrounding Be stars, Menzel case B is expected for hydrogen (i.e. detailed balance between levels 1 and 2; level 2 is the effective ground level; see e.g. Hummer \& Storey 1987). 
However, line fluxes are likely affected by strong optical depth effects, since also the continuum becomes optically thick. At infrared wavelengths transitions from high lying energy levels are found, whose populations are believed to be well described by Local Thermodynamic Equilibrium (LTE) (however, see below).

Theoretically, the line strengths and shapes of $\mathrm{HI}$ recombination lines in Be star disks were investigated by Waters et al. (1992), Zaal et al. (1995), and Marlborough et al. (1997). These studies show that, assuming that the source function is the Planck function at the local electron temperature and density, a relation exists between the observed line flux and line opacity (defined by the parameter $\mathrm{X}_{\text {line }}$ in Zaal et al. (1995)) whose slope is determined by the radial density gradient. The shape of the HI recombination lines is a sensitive probe of the velocity field in the disk. If rotation is important in the inner disk, and expansion dominates the kinematics of the disk in the outer regions, this will result in asymmetric double-peaked line profiles with the blue peak stronger than the red one for the strongest lines, and symmetric lines for the weak lines (Waters et al. 1992). Studies of the infrared spectrum of $\gamma$ Cas have shown that its $\mathrm{HI}$ emission line spectrum is difficult to understand in terms of simple recombination line theory (see e.g. Hamann \& Simon 1987).

\section{ISOPHOT continuum observations}

Several bright Be stars were observed in the guaranteed time of the ISO Science Operations Centre using the ISOPHOT instrument (Lemke et al. 1996). These observations were aimed at filling in the gap in spectral coverage between the IRAS $12-60 \mu \mathrm{m}$ and the sub-mm and radio observations. The observations were performed at $12,25,60,90,135$ and $160 \mu \mathrm{m}$. The 12 and $25 \mu \mathrm{m}$ observations were obtained using triangular chopping with a chopper throw of 90 arcsec, while for the longer wavelength observations a mapping strategy was chosen in order to allow for a more reliable determination of the background. A full description of the observations and the data analysis will be published elsewhere (Trams et al., in preparation). In Figure 1 we plot the Spectral Energy Distribution (SED) of 8 programme stars between 12 and $160 \mu \mathrm{m}$.

We have compared the IRAS $12-60 \mu \mathrm{m}$ data with the ISO photometry, and found that the ISO $25 \mu \mathrm{m}$ fluxes of all targets were significantly weaker than those observed by IRAS. It is unlikely that this is due to variability, given the size of the sample. In addition, the $12 \mu \mathrm{m}$ fluxes for the brightest objects were systematically higher than the IRAS data. No systematic trends could be observed at $60 \mu \mathrm{m}$ (which were taken using the mapping mode). The systematic effects at 12 and $25 \mu \mathrm{m}$ may be due to memory effects of the ISOPHOT P detectors. We decided to ignore the ISO 12 and $25 \mu \mathrm{m}$ data when deriving spectral indices. In Table 1, we compare the IRAS 12-60 $\mu \mathrm{m}$ spectral index $\alpha$, defined as $\mathrm{S}_{\nu} \propto \lambda^{\alpha}$, with the ISO 60-160 $\mu \mathrm{m}$ index. While in most stars the slope of the IRAS and ISO continua are comparable, for $\psi$ Per, $\eta$ Tau, and EW Lac they are rather different.

For the mapping observations, we have carefully inspected the level of the galactic background radiation and its spatial variation near the target stars. We found that the background is usually well behaved and the measured flux levels are reliable. However, for $\psi$ Per we found a steep background gradient of several 


\begin{tabular}{|c|c|c|c|}
\hline \multirow[t]{2}{*}{ Star } & \multirow[t]{2}{*}{ IRAS $(12,25,60)$} & \multicolumn{2}{|c|}{ ISO $(60,90,135,160)$} \\
\hline & & & (2) \\
\hline$\gamma$ Cas & $-1.2 \pm 0.07$ & $-1.4 \pm 0.02$ & $-1.4 \pm 0.3$ \\
\hline$\alpha$ Eri & $-2.0 \pm 0.04$ & $-1.5 \pm 0.05$ & $-1.5 \pm 0.2$ \\
\hline$\phi$ Per & $-1.1 \pm 0.10$ & $-1.2 \pm 0.06$ & $-1.1 \pm 0.3$ \\
\hline$\psi$ Per & $-0.9 \pm 0.05$ & $-0.2 \pm 0.04$ & $-0.4 \pm 0.7$ \\
\hline$\eta \mathrm{Tau}$ & $-1.1 \pm 0.06$ & $-2.5(:)$ & $-2.5(:)$ \\
\hline$\zeta \mathrm{Tau}$ & $-1.2 \pm 0.08$ & $-1.3 \pm 0.03$ & $-1.3 \pm 0.26$ \\
\hline$\chi \mathrm{Oph}$ & $-0.9 \pm 0.1$ & $-0.8 \pm 0.02$ & $-0.7 \pm 0.18$ \\
\hline EW Lac & $-0.9 \pm 0.1$ & $-0.3 \pm 0.09$ & $-0.2 \pm 0.55$ \\
\hline
\end{tabular}

Table 1. Power law indices for the 8 programme stars derived from the IRAS and ISO data. The indices were calculated by fitting a powerlaw spectrum to the IRAS 12,25 and 60 micron points (second column), and to the ISO $60,90,135$ and 160 micron points (third and fourth column). The different indices for the ISO fits are for fits where only the source flux uncertainty was taken into account (column (1)) and for fits where also the background standard deviation (i.e. background structure) was taken into account (column (2)); For $\eta$ Tau the slope is based on 60 and $90 \mu \mathrm{m}$ data, and has large error bars.

Jy over the $270 \times 270$ arcsec map taken with the C200 detector, without a clear point source signal. We conclude that the long wavelength measurements of $\psi$ Per are unreliable.

The spectral slope of $\eta$ Tau between 60 and $90 \mu \mathrm{m}$ is very steep but with large error bars. However, the 135 and $160 \mu \mathrm{m}$ points are upper limits that are a factor of 3 lower than expected on the basis of the short-wavelength IRAS and ISO data. Sub-mm observations taken in 1989 showed flux levels consistent with those expected from extrapolation of the IRAS fluxes, and a turnover at radio frequencies (Waters et al. 1991). The ISO data suggest a significant change in the long-wavelength spectrum of $\eta$ Tau, with a turnover at about $60 \mu \mathrm{m}$. Such a change is not expected given the lack of strong variability in e.g. the $\mathrm{H} \alpha$ line (Coté \& Waters 1987).

The flat ISO spectral index of EW Lac is remarkable and unexpected since it is contrary to the trend of a spectral steepening observed in Be stars at millimeter and radio wavelengths (see above). The origin of the high $90-160 \mu \mathrm{m}$ fluxes may be related to the presence of cold dust near the star, or due to free-free emission from ionized gas at large distance from the star. If the flat spectral index is caused by cold dust, then for reasonable estimates of dust temperature and dust emissivity, the size of the object would be much larger than observed. Only if we adopt large grains that radiate as black bodies at $100 \mu \mathrm{m}$ are the angular size estimates consistent with the point-like nature of the ISO sources. The origin of such large grains remains unclear however. It is unlikely that such grains were formed in the ionized inner disk. They may be remnants of the star formation process. If the flat spectral index is due to free-free emission, this would imply that an additional low density component with large emission measure begins 


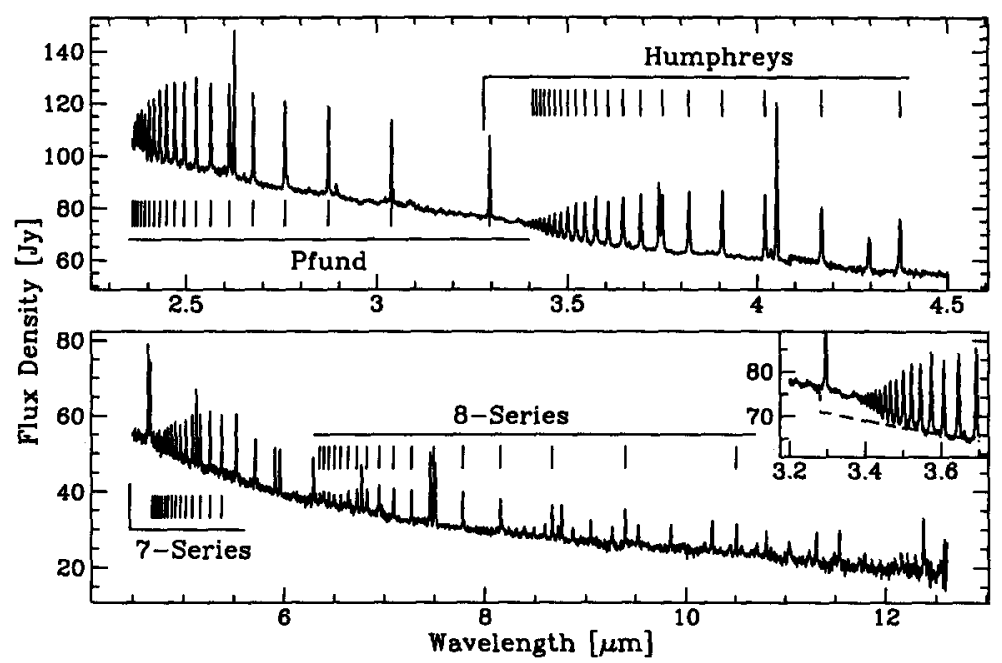

Figure 2. SWS AOT01 speed 4 spectrum of $\gamma$ Cas between 2 and $12 \mu \mathrm{m}$ (Hony et al. 2000). The spectrum is dominated by numerous emission lines from hydrogen. A few He lines are also seen. Notice the spectral jump near $3.4 \mu \mathrm{m}$.

to contribute to the total flux near $100 \mu \mathrm{m}$. This could be caused by a detached ring of gas, perhaps resulting from variable mass loss through the disk. We note that EW Lac is known to show variations at near-IR and radio wavelengths (Dougherty \& Taylor 1994; Dougherty et al. 1991). Unpublished sub-mm data we have taken also show evidence for variations at $850 \mu \mathrm{m}$.

In the case of $\alpha$ Eri the flattening of the spectral index between IRAS and ISO wavelength ranges can be explained as the onset of the continuum freefree excess near $90 \mu \mathrm{m}$. This onset occurs at much shorter (near-IR or optical) wavelengths in most $\mathrm{Be}$ stars due to the higher densities in these disks.

\section{ISO-SWS observations}

For a small number of stars, spectra were obtained with the Short Wavelength Spectrograph (SWS; de Graauw et al. 1996) in the guaranteed time of the SWS consortium. In most cases spectral line scans (AOT02) of HI, HeI and [FeII] lines were obtained, while for $\delta$ Cen and $\gamma$ Cas full scans at reduced spectral resolution (AOT01 speed 4) were taken. In addition, for about 1 month after helium boil-off of the ISO cryostat, the SWS instrument was able to take 2.4-4 $\mu \mathrm{m}$ spectra of some 250 stars, among which were several Be stars. Below we discuss the ISO-SWS spectrum of $\gamma$ Cas, and some examples of the "post-helium boil off" spectra. 


\section{1. $\gamma$ Cas}

The ISO-SWS spectrum of $\gamma$ Cas between 2.4 and $12 \mu \mathrm{m}$ is shown in Figure 2 (Hony et al. 2000) and is characterized by many strong lines of HI. Very few lines of HeI are seen, indicating that the bulk of the gas in the disk is cool $\left(\mathrm{T} \approx 10^{4} \mathrm{~K}\right)$. There is no sign of forbidden line emission of singly ionized metals, such as [NiII] or [FeII]. This is in marked contrast with stars with dense, spherically symmetric winds such as P Cygni (Lamers et al. 1996). Clearly an extended, low-density region of gas with modest ionisation is absent in $\gamma$ Cas. The 8-13 $\mu \mathrm{m}$ spectrum of $\gamma$ Cas shown by Rinehart et al. (2000) agrees well with the SWS data.

The 2.4-20 $\mu \mathrm{m}$ continuum of $\gamma$ Cas is well represented by a power law $S_{\nu} \propto \nu^{\alpha}$ with $\alpha=1.0 \pm 0.03$. This is slightly flatter than the spectral slope derived from IRAS photometry (Waters et al. 1987). For an isothermal disk with geometry as assumed by Waters (1986) this corresponds to a radial density gradient $\rho(\mathrm{r}) \propto \mathrm{r}^{-n}$ with $\mathrm{n}=2.8$. The ISOPHOT data for $\gamma$ Cas between 60 and $160 \mu \mathrm{m}$ (Figure 4) are significantly below the extrapolation of the ISOSWS spectrum to longer wavelengths. It is unlikely that this is due to a poor background subtraction in the ISOPHOT maps, since this would tend to overestimate the flux. The change in slope of the continuum energy distribution of $\gamma$ Cas was was already noted by Waters et al. (1991) on the basis of IRAS and millimeter photometry. It suggests either less ionized material at large distance from the star, or a change in geometry.

The spectrum of $\gamma$ Cas shows a jump in flux level at $3.4 \mu \mathrm{m}$, corresponding to the Humphreys jump $(6-\infty)$. This jump, observed in emission, is similar to the Balmer jump at optical wavelengths, and can be used to determine the electron temperature in the disk. This is because the bound-free gaunt factor, and thus the opacity, depends on temperature. The opacity suddenly increases when going across the jump from long to short wavelength. This causes a change in the effective radiating surface and therefore in flux level. The size of the jump is determined by the jump in opacity and thus depends on temperature. Hony et al. (2000) find about $9500 \mathrm{~K}$ for $\gamma \mathrm{Cas}$, in good agreement with the volumeaveraged temperature derived by Millar et al. (1998; see also Millar, these proc.) from model calculations. The disk temperature is significantly lower than the effective temperature (Telting et al. 1993), and explains the lack of significant HeI line emission.

The line strengths of the Pfund, Humphreys, Hansen-Strong, lower level 8 and 9 series of $\gamma$ Cas are plotted against line strength in Figure 4. Also shown are the line widths (full width half maximum). All lines are much fainter than predicted on the basis of optically thin case B recombination line calculations and assuming an emission measure for the disk derived from the continuum. This demonstrates that all lines are optically thick, which is not surprising given the fact that the continuum is also partially optically thick. The line widths decrease with increasing line strength for all observed series. This is consistent with line radiation emerging from a rotating disk. We note that the maximum line width of the HI observed in $\gamma$ Cas exceeds $2 \times$ vsini $\left(=2 \times 230 \mathrm{~km} \mathrm{~s}^{-1}\right.$, Slettebak 1982), suggesting that the inner disk rotates faster than the stellar photosphere. This effect was previously noted for a larger sample of stars by Hanuschik (1987), using optical permitted FeII lines. 


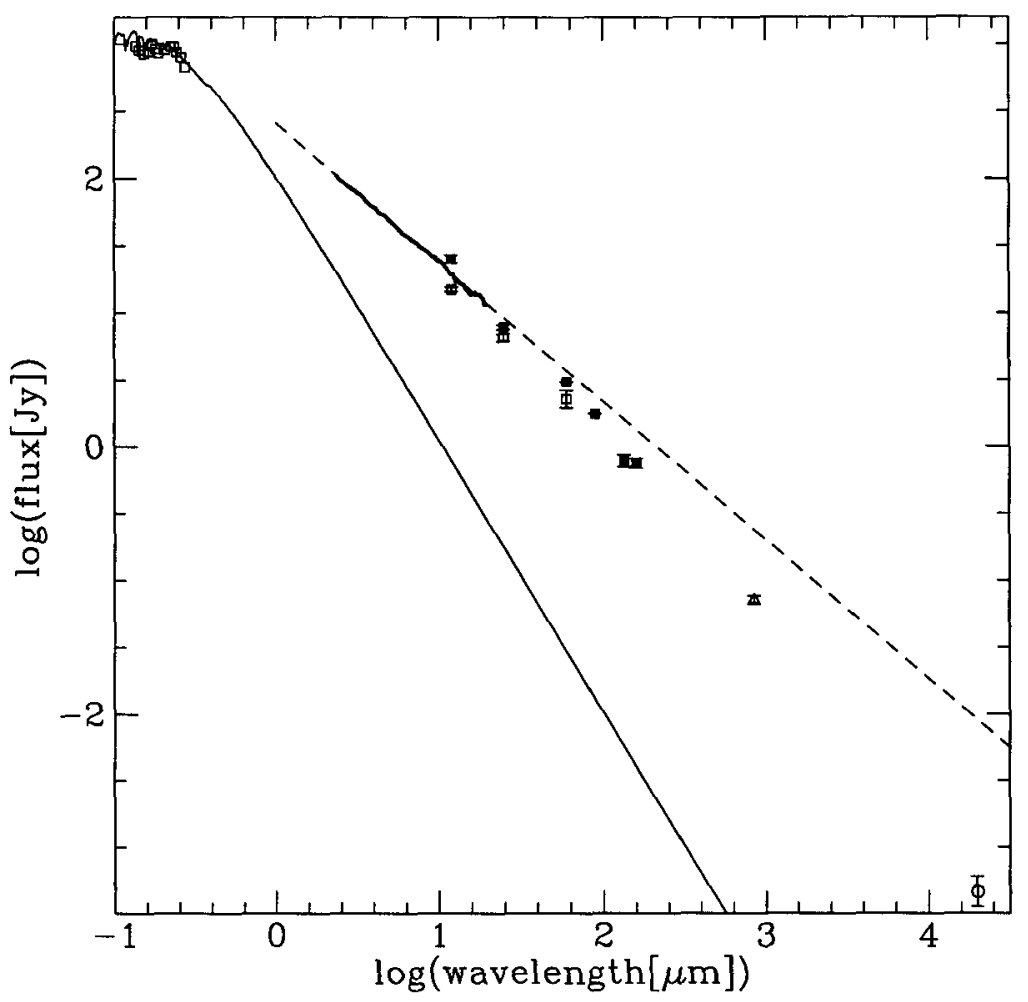

Figure 3. Observed UV to IR energy distribution of $\gamma$ Cas. UV and optical data were taken from Telting et al. (1993). Also included are the ISO-SWS spectrum and the ISO-PHOT measurements between 60 and $160 \mu \mathrm{m}$. Millimeter and radio data are taken from Waters et al. (1991).

It is important to realize that the observed line radiation emerges from those parts of the disk that are optically thin in the continuum. From the line widths of the weakest lines of the Humphreys and Hansen-Strong series, that exceed $500 \mathrm{~km} / \mathrm{s}$, we can deduce that these lines must be formed in the innermost 2-3 $\mathrm{R}_{*}$ of the disk (unless the rotation in the disk is highly non-Keplerian). These weak lines are at a wavelength where the local continuum is optically thick at 2-3 $R_{*}$, and no line emission would be expected unless the source function in the line exceeds that of the local continuum. The observations are consistent with a surface layer of increased temperatures (up to 30 per cent above the bulk of the disk, i.e. $\sim 13000 \mathrm{~K}$ ).

The observed flux from $\mathrm{HI}$ lines of intermediate upper levels (typically upper level 10-15) are observed to be independent of intrinsic line strength. This is contrary to what is expected if the lines are formed in a large disk and assuming LTE. Apparently, the outer regions do not contribute significantly to the line flux, since also the line widths are similar for all lines from upper level 


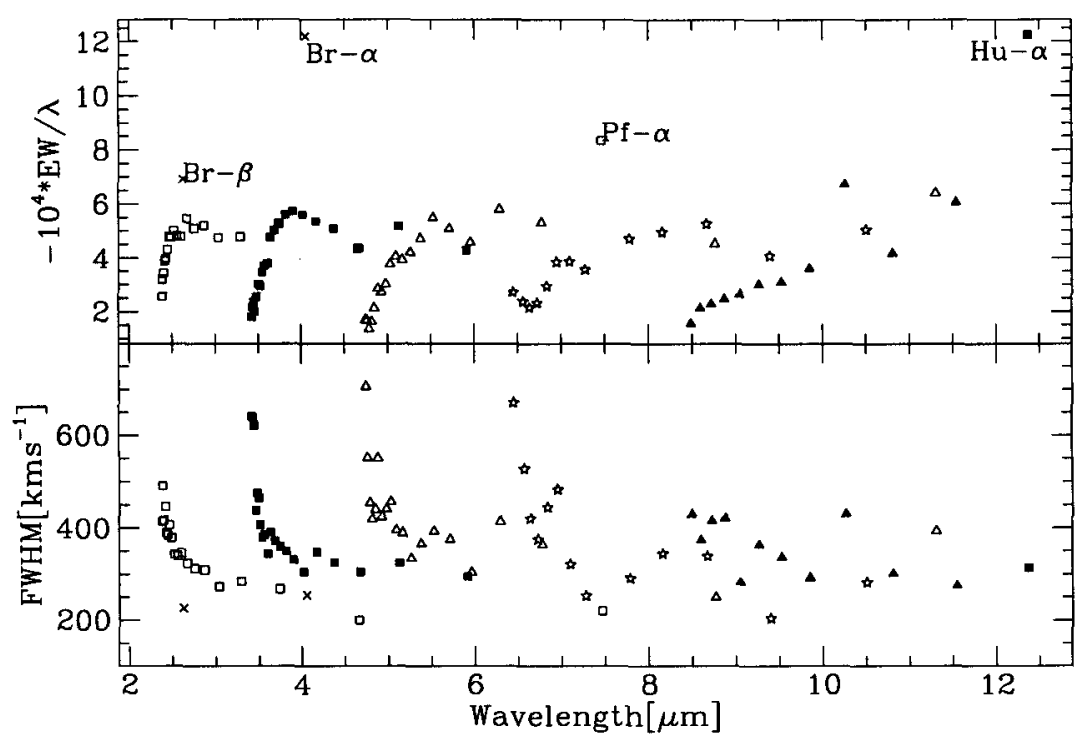

Figure 4. Top panel: $\mathrm{EW} / \lambda$ of the HI lines in the ISO-SWS spectrum of $\gamma$ Cas as a function of wavelength. Open squares are Pfund series, filled squares are Humphreys series, open triangles are Hansen-Strong series, stars are lower level 8 series, and filled triangles are lower level 9 series. The EWs have been determined with respect to the local continuum. Bottom panel: Observed Full Width at Half Maximum of the HI lines versus wavelength. The symbols refer to the same series as in the upper panel. The lines become narrower with increasing line strength as expected in a rapidly rotating disc in which the rotational velocity decreases with distance.

10-15, irrespective of lower level and intrinsic line strength. The observations suggest that the local continuum determines the line strength. It is unlikely that the observed effect is due to a lack of material in the outer regions, since the continuum energy distribution demonstrates that material emitting free-free radiation is present to large distance from the star, although there is a change in the slope of the continuum spectrum near $60 \mu \mathrm{m}$ (Figure 3) It is possible that the line source function of the intermediate levels is suppressed in the outer regions, perhaps due to a cascade to lower levels in the outer disk. Detailed 2dimensional, non-LTE multi-level calculations are needed to determine the cause of these remarkable line strengths.

\subsection{Other Be stars}

In Figure 5 we show the 3.8-4.1 $\mu \mathrm{m}$ spectrum of six stars, among which $\gamma$ Cas and the hypergiant $\mathrm{P}$ Cygni, which presumably has a spherically symmetric outflow. The other spectra shown in Figure 4 were taken in the post-helium 

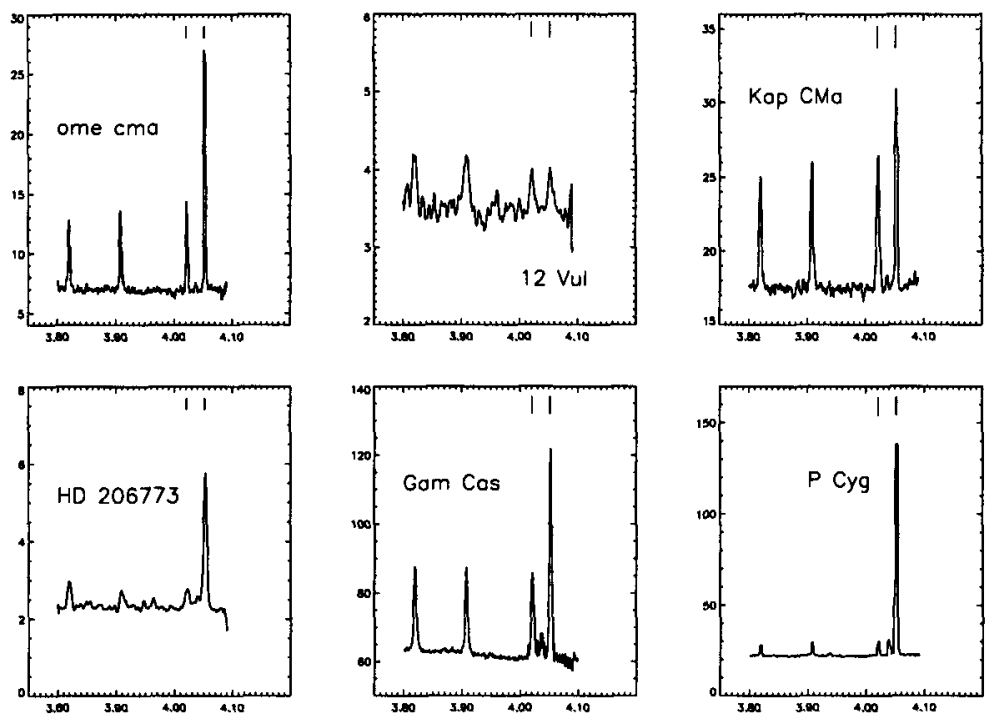

Figure 5. SWS spectra of several Be stars in the 3.8-4.1 $\mu \mathrm{m}$ range, showing remarkable values for the line flux ratio $\operatorname{Br} \alpha(4.052 \mu \mathrm{m})$ to HI 14-6 $(4.0209 \mu \mathrm{m})$, indicated by tick marks. This suggests high line optical depth. The hypergiant $\mathrm{P}$ Cygni is shown for comparison. Line flux ratios may be used to identify Be stars at infrared wavelengths.

boil off programme. The HI line flux ratios show large star to star variations. In particular the ratio $\operatorname{Br} \alpha(4.052 \mu \mathrm{m})$ to $\mathrm{HI} 14-6(\lambda 4.0209 \mu \mathrm{m})$ is illustrative, and we limit the discussion to these two lines. In $\mathrm{P}$ Cygni, the line flux ratio $\mathrm{Br} \alpha / \mathrm{HI} 14-6$ is consistent with that expected from the intrinsic line strengths, taking into account optical depth effects. For the Be stars however, this ratio is usually much smaller, and in $12 \mathrm{Vul}$ it is even close to unity. For $\gamma$ Cas this ratio is in between the values found for $\mathrm{P}$ Cygni and 12 Vul. It is likely that a similar mechanism as in $\gamma$ Cas is responsible for the low $\mathrm{Br} \alpha / \mathrm{HI14}-6$ ratio in the other Be stars, and that this effect is typical for the infrared spectra of Be stars. Low infrared line flux ratios of certain HI recombination lines may therefore be an interesting way to detect high-density circumstellar disks in hot stars, if other information (e.g. high spectral resolution or data at other wavelengths) is lacking. Interestingly, for the star $\alpha$ Eri both low and higher values of the $\mathrm{Br} \alpha / \mathrm{HI} 14-6$ ratio are observed, indicating variability in the excitation conditions and/or densities in the disk.

Acknowledgments. LBFMW acknowledges financial support from an NWO Pionier grant. 


\section{References}

Ashok, N.M., Bhatt, H.C., Kulkarni, P.V., et al. 1984, MNRAS, 211, 471

Bjorkman, J., Cassinelli, J.P. 1993, ApJ, 409, 429

Cassinelli, J.P., Hartmann, L. 1979 ApJ, 212, 488

Chalabaev, A.A., Maillard, J.P. 1985, ApJ, 294, 640

Coté, J., Waters, L.B.F.M. 1987, A\&A, 176, 93

Dachs, J., Wamsteker, W. 1982, A\&A, 107, 240

Dachs, J., Hanuschik, R., Kaiser, D., Rohe, D. 1986, A\&A, 159, 276

Dougherty, S.M., Taylor, A.R. 1992, Nature 359, 808

Dougherty, S.M., Taylor, A.R. 1994, MNRAS, 2691123

Dougherty, S.M., Waters, L.B.F.M., Burki, et al. 1994, A\&A, 290, 609

Gehrz, R.D., Hackwell, J.A., Jones, T.W. 1974, ApJ, 191, 675

Hamann, F., Simon, M. 1987, ApJ, 318356

Hanuschik, R.W. 1987, A\&A, 173, 299

Hony, S., Waters, L.B.F.M., Zaal, P.A. et al. 2000, A\&A, in press

Hummer, D.G., Storey, P.J. 1987, MNRAS, 224, 801

De Graauw, Th., et al. 1996, A\&A, 315, L49

Kessler, M.F., et al. 1996, A\&A, 315, L27

Lamers, H.J.G.L.M., Najarro, P., Kudritzki, R.P., et al. 1996, A\&A, 315, L229

Lemke, D., et al. 1996 A\&A, 315, L64

Marlborough, J.M., Zijlstra, J.-W., Waters, L.B.F.M. 1997, A\&A, 321, 867

McLean, I.S., Brown, J.D. 1978 A\&A, 69, 291

Millar, C.E., Marlborough, J.M. 1998, ApJ, 494, 715

Persson, S.E., McGregor, P.J. 1985, AJ, 90, 1860

Quirrenbach, A., Bjorkman, K.S., Bjorkman, et al. 1997, ApJ, 479, 477

Rinehart, S.A., Houck, J.R., Smith, J.D. 2000, AJ, in press

Sellgren, K., Smith, R.G. 1992, ApJ, 388, 178

Slettebak, A. 1982, ApJS, 50, 55

Stee, Ph., De Araujo, F.X., Vakili, F., Mourard, D., et al. 1995, A\&A, 300, 219

Stee, Ph., Vakili, F., Bonneau, D., Mourard, D. 1998, A\&A, 332, 268

Taylor, A.R., Waters, L.B.F.M., Bjorkman, K.S., Dougherty, S.M. 1990, A\&A, 231,453

Telting, J., Waters, L.B.F.M., Persi, P., Dunlop, S.R. 1993, A\&A, 270, 355

Waters, L.B.F.M. 1986, A\&A, 162, 121

Waters, L.B.F.M., Coté, J., Lamers, H.J.G.L.M. 1987, A\&A, 176, 93

Waters, L.B.F.M., van der Veen, W.E.C.J., Taylor, A.R., et al. 1991, A\&A, 244, 120

Waters, L.B.F.M., Marlborough, J.M. 1992, A\&A, 253, L25

Wright, A.E., Barlow, M.J. 1975, MNRAS, 170, 41

Zaal, P.A., Waters, L.B.F.M., Marlborough, J.M. 1995 A\&A, 299, 574 